\title{
Bone Morphogenic Protein Is a Viable Adjunct for Fusion in Minimally Invasive Transforaminal Lumbar Interbody Fusion
}

\author{
M Mashfiqul Arafin Siddiqui ${ }^{1}$, Ana Rosario P. Sta.Ana ${ }^{1}$, William Yeo ${ }^{2}$, Wai-Mun Yue ${ }^{1}$ \\ ${ }^{1}$ Department of Orthopaedic Surgery, Singapore General Hospital, Singapore \\ ${ }^{2}$ Orthopaedic Diagnostic Centre, Singapore General Hospital, Singapore
}

Study Design: Comparison of prospectively collected data of patients undergoing minimally invasive surgery transforaminal lumbar interbody fusion (MIS-TLIF) with and without recombinant human bone morphogenic protein 2 (BMP).

Purpose: To compare the clinical, radiological outcome and complications of patients undergoing MIS-TLIF with and without BMP. Overview of Literature: BMP is an effective fusion enhancer with potential complications. Direct comparison of MIS-TLIF with and without BMP is limited to retrospective studies with short follow-up.

Methods: From June 2005 to February 2011, consecutive cases of MIS-TLIF performed by a single surgeon were included. North American Spine Society (NASS) score, Oswestry disability index (ODI), Short Form-36 (SF-36), and visual analogue score (VAS) were assessed preoperatively and at 6 and 24 months postoperatively. Fusion rates and complications were noted.

Results: The 252 cases comprised 104 non-BMP and 148 BMP cases. The BMP group was significantly older (mean age, 60.2 vs. 53.9; $p<0.01$ ). Preoperative scores were similar. Immediate postoperative morphine usage was significantly lower in the BMP group (12.4 mg vs. $20.1 \mathrm{mg}, p<0.01$ ). At 6 months, the BMP group had lower VAS back and leg pain scores $(p<0.01)$. At 2 years, the BMP group had better leg pain scores ( $p<0.01)$, ODI (15.4 vs. 20.3, $p=0.04$ ) and NASS scores ( 8.8 vs. $15.8, p<0.01)$ ). Both groups showed significant clinical improvement compared to their preoperative levels. The BMP group attained a significantly higher rate of fusion at 6 months follow-up (88.4\% vs. $76.8 \%, p=0.016$ ) with no difference at 2 years. The non-BMP and BMP group had $12(11.5 \%)$ and $9(6.1 \%)$ complications and $5(4.8 \%)$ and $2(1.4 \%)$ reoperations, respectively.

Conclusions: The use of BMP to augment fusion in MIS-TLIF is an acceptable alternative that has potential benefits of less pain in early and intermediate postoperative follow-up.

Keywords: Lumbar spine; Degenerative; Transforaminal interbody fusion; Bone morphogenic protein; TLIF BMP

\section{Introduction}

Fusion of the lumbar spine is the surgical treatment of choice for a number of spinal disorders, including degenerative, traumatic, and neoplastic disorders. The use of minimally invasive surgery (MIS) techniques to perform these fusions has been well described. These techniques reduce tissue trauma, blood loss, and postoperative pain and enable quicker patient recovery [1-5].

A disadvantage with the MIS approach is the frequent

Received Jan 18, 2016; Revised Apr 20, 2016; Accepted May 7, 2016

Corresponding author: Wai-Mun Yue

Department of Orthopaedic Surgery, Singapore General Hospital, Outram Road, 169608 Singapore

Tel: +65-6321-4603, Fax: +65-6224-8100, E-mail: yuewm@singnet.com.sg 
paucity of local bone graft required for fusion. Iliac crest bone graft is the gold standard for stimulating arthrodesis of the spine. This graft provides osteogenic cells, osteoinductive factors and an osteoconductive scaffold needed for bone regeneration. The problem with harvesting iliac crest bone graft is the significant donor site morbidity and postoperative complications including infection, hematoma, intractable pain, fracture, and neurovascular injury [6-8]. Bone graft substitutes as well as bone morphogenic protein (BMP) have been used to in a bid to avoid some of these complications. BMP, particularly recombinant human BMP-2 (rhBMP-2), has significant osteoinductive properties. While the use of rhBMP- 2 often improves the rate of bony fusion, there is concern that rhBMP-2 can creating new iatrogenic and potentially symptomatic problems for the patient $[1,7,9-16]$. A study by the Yale University Open Data Access (YODA) Project noted that while rhBMP-2 improves rate of fusion compared to autogenous iliac crest graft and improves back pain and quality of life between 6 and 24 months after surgery, it may also increase the risk for heterotopic bone formation, osteolysis, radiculitis, and retrograde ejaculation, and may have a possible association with an increased risk for cancer [9].

In this study, we compare the clinical and radiological outcomes, and complications of MIS transforaminal lumbar interbody fusion (TLIF) performed with and without the use of rhBMP-2.

\section{Materials and Methods}

This study was approved by our Institutional Review Board (IRB number 2013/971/D). All consecutive patients who had a MIS-TLIF performed in our academic tertiary hospital by a single surgeon from June 2005 to February 2011 with minimum 2-year follow-up were identified from our spine registry and were considered inclusion. Patients were included regardless of the number of levels fused. Patients who had previous spinal instrumentation, tumor or neoplastic spinal pathologies, spinal infections and acute spinal trauma were excluded.

Patients were divided into two groups: MIS-TLIF performed with and without the use of rhBMP-2 (BMP and non-BMP, respectively). Local bone graft, autogenous iliac bone graft, and demineralized bone matrix (OSTEOFIL DBM; Medtronic, Minneapolis, MN, USA), which are routine and acceptable in cases of MIS-TLIF, were used in the non-BMP group. Local bone graft was used together with rhBMP-2 (INFUSE; Medtronic) as fusion enhancers in the BMP group. Patients were assigned to the group based on their preference preoperatively after a detailed discussion and their informed consent.

All patients had a similar surgical technique. A unilateral facetectomy with tubes was done followed by a discectomy and endplate preparation. For the BMP group, one small kit of BMP $(4.2 \mathrm{mg})$ sponge was inserted into the anterior disc space followed by local bone graft (if sufficient). A unilateral cage filled with local bone graft was inserted last. No additional allograft was inserted for this group. For the non-BMP group, the aforementioned substitutes were used in place of BMP. Decompression laminectomy of the ipsilateral side, internal laminoplasty of the contralateral side followed by bilateral percutaneous posterior instrumentation was then performed.

Patient demographics were noted. Clinical outcomes were measured using North American Spine Society (NASS) scores for back pain/disability and neurogenic symptoms, Oswestry disability index (ODI), Short Form36 (SF-36) scores, and visual analogue scale (VAS) for back and leg pain. Independent assessors performed each patient assessment pre-surgery, at 6 months and 2 years after surgery. The development of any erectile dysfunction (ED) among sexually active male patients was assessed using the International Index of Erectile Function (IIEF-5) questionnaire. All outcome data was collected prospectively.

Immediate postoperative pain was assessed based on the amount of postoperative analgesia administered. Morphine $(\mathrm{mg})$ was the standard unit measured. Conversion of pethidine to morphine-equivalent doses was done for patients who received intramuscular pethidine instead of intravenous patient controlled anesthesia morphine (using the formula $1 \mathrm{mg}$ morphine IV: $7.5 \mathrm{mg}$ pethidine intramuscular/intravenous).

Two independent assessors using static and dynamic radiographs taken at 6 months and 2 years postsurgery evaluated fusion rates based on Bridwell criteria [17]. All surgeries were performed by a single surgeon according to the technique described previously $[2,5,18]$. Graft materials were placed anteriorly and contralateral to the annulotomy within the interbody space and packed into the interbody cage.

Statistical analysis was performed with SPSS ver. 17.0 (SPSS Inc., Chicago, IL, USA). The $\chi^{2}$ contingency table 
Table 1. Demographics and perioperative data

\begin{tabular}{|c|c|c|c|}
\hline Variable & Non-BMP & BMP & $p$-value \\
\hline No. of patients & 104 & 148 & \\
\hline \multicolumn{4}{|l|}{ Follow-up rate } \\
\hline At 6 mo & $98(94.2)$ & $144(97.3)$ & \\
\hline At $2 \mathrm{yr}$ & 95 (91.3) & $129(87.2)$ & \\
\hline Sex & & & 0.913 \\
\hline Male & $33(31.7)$ & $46(31.1)$ & \\
\hline Female & $71(68.3)$ & $102(68.9)$ & \\
\hline Age (yr) & 53.9 & 60.2 & $<0.01$ \\
\hline $\mathrm{BMI}$ & 25.6 & 25.3 & 0.634 \\
\hline No. of levels & & & $<0.01$ \\
\hline 1 & $94(90.4)$ & $104(70.3)$ & \\
\hline 2 & $10(9.6)$ & $43(29.1)$ & \\
\hline 3 & 0 & $1(0.6)$ & \\
\hline Presence of spondylolisthesis & $65(62.5)$ & $82(55.4)$ & 0.261 \\
\hline Mean duration of operation (min) & 164 & 158 & 0.312 \\
\hline Mean length of stay (day) & 3.5 & 3.2 & 0.389 \\
\hline Mean time to return to work (day) & 83.8 & 93.6 & 0.601 \\
\hline Mean morphine use (mg) & 20.1 & 12.4 & $<0.01$ \\
\hline Mean days to ambulation & 1.1 & 1.2 & 0.165 \\
\hline
\end{tabular}

Values are presented as number (\%).

$\mathrm{BMP}$, bone morphogenic protein; BMI, body mass index.

was used to compare categorical data (gender, fusion grading at 6 months and 2 years postsurgery, return to full function, patient rating of the overall result of surgery, and complications). Student's $t$-test for independent samples was used to compare continuous variables (age, body mass index [BMI], length of operation, and length of stay). Tests of between-subject effects were used to evaluate the differences in VAS, ODI, SF-36, and NASS. In all analyses, significance was defined as $p<0.05$.

\section{Results}

A total of 252 consecutive eligible patients were included, with 104 in the non-BMP group and 148 in the BMP group. The overall follow-up rate at 6 months and 2 years was $96 \%$ and $88.9 \%$, respectively. Patients who did not return for follow-up were mainly residents of another country who had returned to their home country following surgery. One patient in each group died from unrelated causes.

The mean age of patients in the BMP group was signifi- cantly higher than the non-BMP group (60.3 years vs. 53.9 years, $p<0.01)$. Patients in the BMP group also had significantly more patients with multi-level surgery compared to the non-BMP group $(29.7 \%$ vs. $9.6 \%, p<0.01)$. Gender distribution and BMI were similar between the groups. Patients in the BMP group had significantly lower morphine usage compared to patients who did not use BMP (12.4 $\mathrm{mg}$ vs. $20.1 \mathrm{mg}, p<0.01)$. Perioperative measurements of duration of operation, length of stay, and days to return to work were not significantly different between the groups. Table 1 summarizes patient's demographics and perioperative data.

Table 2 presents the preoperative clinical scores and their change at 6 months and 2 years. Preoperative VAS scores for back and leg pain, ODI, SF-36, NASS scores, and IIEF scores were similar. At 6 months, patients in the BMP group had lower back and leg pain scores $(p<0.01)$ compared to patients in the non-BMP group though both groups had statistically significant improvement in their back and leg pain scores compared to their preoperative levels $(p<0.01)$. At 2 years, patients in the BMP group 
Table 2. Clinical scores of patients (preoperative, at 6 months, and at 2 years)

\begin{tabular}{|c|c|c|c|c|c|c|c|c|c|}
\hline \multirow{2}{*}{ Clinical outcome } & \multicolumn{3}{|c|}{ Preoperative (mean) } & \multicolumn{3}{|c|}{6 Months (mean) } & \multicolumn{3}{|c|}{2 Years (mean) } \\
\hline & Non-BMP & BMP & $p$-value & Non-BMP & BMP & $p$-value & Non-BMP & BMP & $p$-value \\
\hline VAS back pain & 6.3 & 5.9 & 0.29 & 2.7 & 1.7 & $<0.01$ & 2.0 & 1.5 & 0.13 \\
\hline VAS leg pain & 6.0 & 5.7 & 0.49 & 1.8 & 0.8 & $<0.01$ & 1.4 & 0.6 & $<0.01$ \\
\hline ODI & 49.2 & 48.3 & 0.72 & 23.9 & 21.0 & 0.20 & 20.3 & 15.4 & 0.04 \\
\hline NASS score & 51.3 & 49.1 & 0.49 & 17.5 & 13.4 & 0.12 & 15.8 & 8.8 & $<0.01$ \\
\hline SF-36 physical function & 41.3 & 41.4 & 0.99 & 64.3 & 65.6 & 0.68 & 68.6 & 73.2 & 0.17 \\
\hline SF-36 role function & 20.4 & 18.9 & 0.68 & 50.3 & 47.2 & 0.60 & 62.6 & 61.8 & 0.89 \\
\hline SF-36 bodily pain & 26.6 & 31.1 & 0.12 & 53.0 & 58.3 & 0.13 & 59.0 & 65.4 & 0.08 \\
\hline SF-36 general health & 62.4 & 63.7 & 0.68 & 63.2 & 65.2 & 0.51 & 66.9 & 70.6 & 0.20 \\
\hline SF-36 vitality & 58.6 & 58.7 & 0.97 & 61.9 & 66.7 & 0.12 & 65.2 & 69.3 & 0.17 \\
\hline SF-36 social functioning & 47.0 & 51.5 & 0.35 & 74.9 & 73.8 & 0.80 & 80.5 & 85.9 & 0.18 \\
\hline SF-36 role functioning & 71.8 & 74.3 & 0.64 & 74.5 & 84.3 & 0.05 & 82.5 & 88.6 & 0.16 \\
\hline SF-36 mental health & 68.2 & 71.2 & 0.27 & 73.3 & 79.2 & 0.02 & 75.8 & 82.3 & $<0.01$ \\
\hline SF-36 average & 49.5 & 51.3 & 0.46 & 64.4 & 67.5 & 0.27 & 70.1 & 74.6 & 0.10 \\
\hline IIEF score & 20.4 & 21.0 & 0.76 & 20.5 & 20.5 & 1.00 & 21.0 & 20.7 & 0.89 \\
\hline
\end{tabular}

BMP, bone morphogenic protein; VAS, visual analogue score; ODI, Oswestry disability index; NASS, North American Spine Society; SF-36, Short Form-36; IIEF, International Index of Erectile Function.
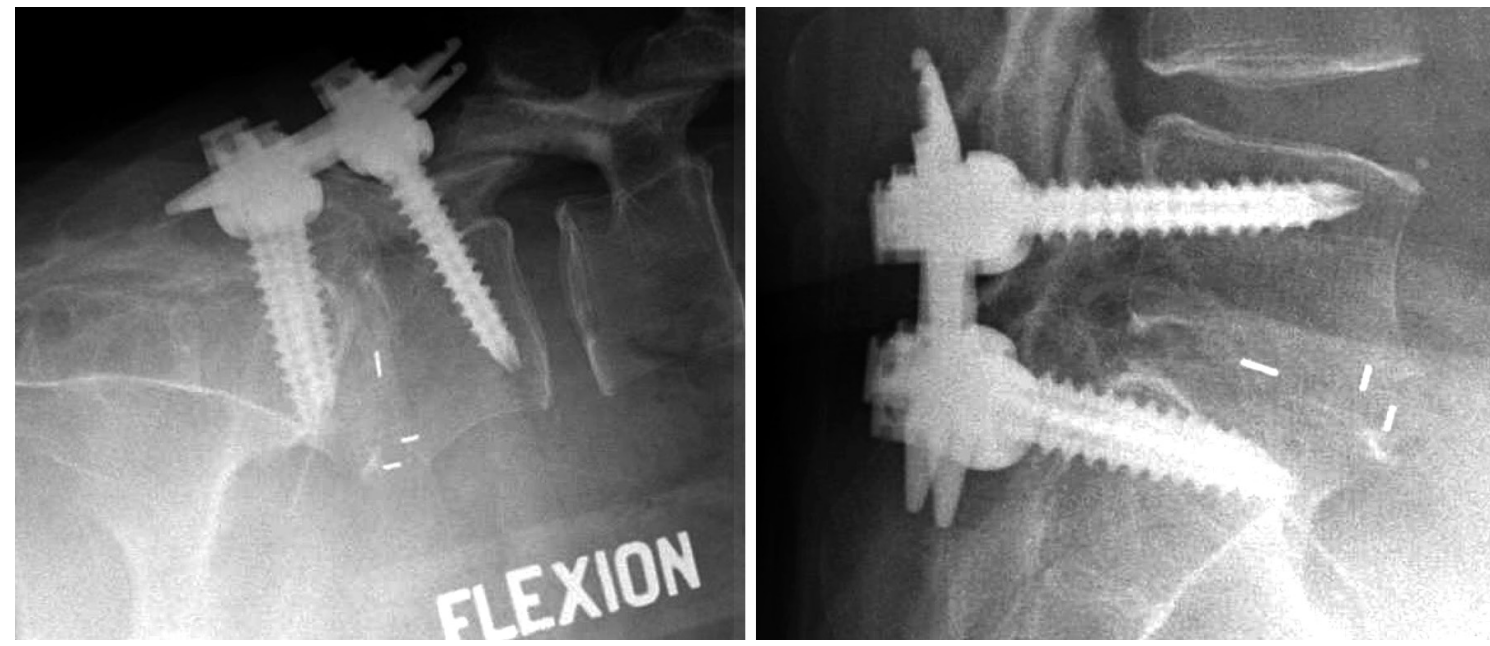

Fig. 1. Erect flexion/extension X-rays of a patient with bone morphogenic protein demonstrating Bridwell grade 1 fusion with no movement.

continued to have better VAS leg pain scores compared to the non-BMP group $(p<0.01)$, better ODI scores $(15.4$ vs. $20.3, p=0.04$ ), and NASS score ( 8.8 vs. $15.8, p<0.01)$. In terms of function, SF-36 scores improved from their preoperative levels in both groups with only minor differences between the groups at 6 months and 2 years.

There was no significant difference between the proportions of patients achieving premorbid functional status at both 6 months (non-BMP, 38.9\%; BMP 43.9\%; $p=0.438$ ) and 2 years (non-BMP, 60.5\%; BMP, 60.0\%; $p=0.944$ ). There was also no difference in patient satisfaction with surgery at 6 months and 2 years (Fig. 1). At 6 months, a good to excellent satisfaction rating was given by $76.1 \%$ of BMP and $81.2 \%$ of the non-BMP patients $(p=0.60)$. At years, $85 \%$ in the BMP group achieved good to excellent satisfaction compared to $77 \%$ in the non-BMP group 

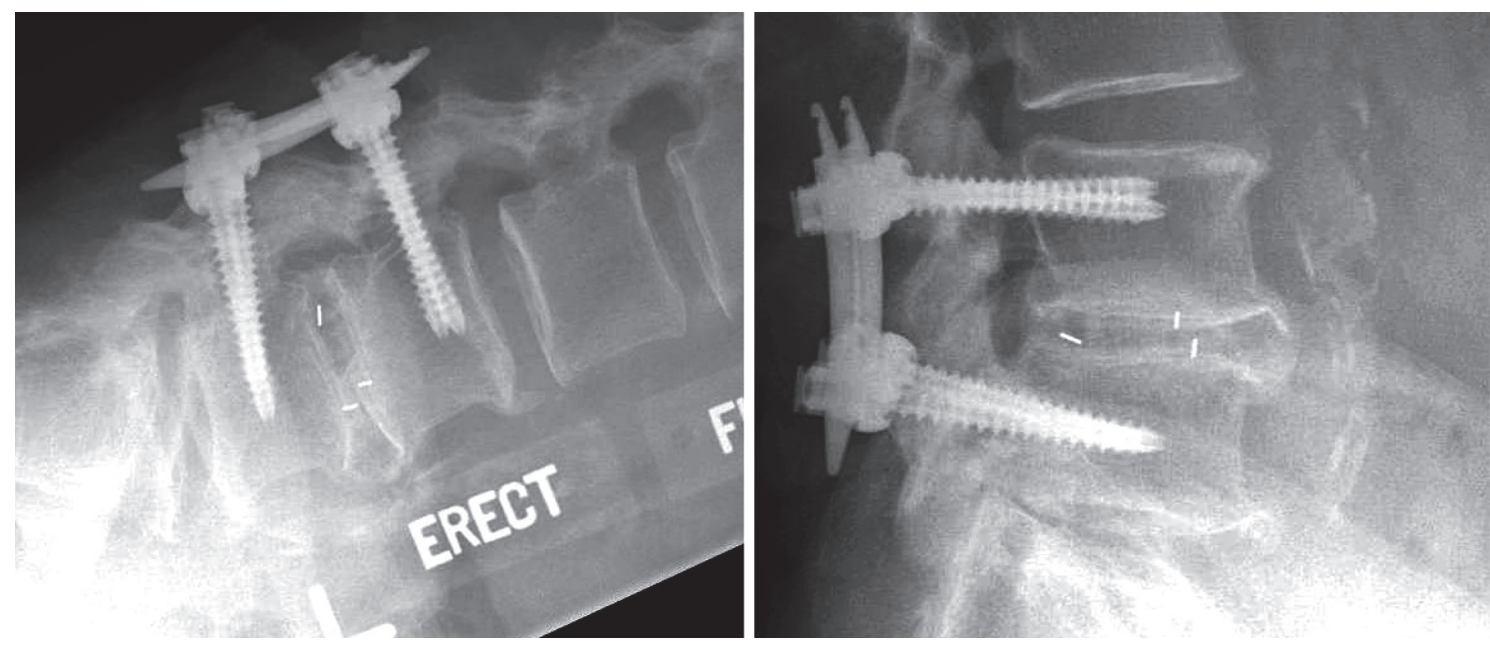

Fig. 2. Erect flexion/extension X-rays of a patient without bone morphogenic protein demonstrating Bridwell grade 1 fusion with no movement.

Table 3. Bridwell grading at 6 and 24 months

\begin{tabular}{|c|c|c|c|c|}
\hline \multirow{2}{*}{ Time } & \multicolumn{4}{|c|}{ Bridwell grade at 6 months } \\
\hline & 1 & 2 & 3 & 4 \\
\hline \multicolumn{5}{|l|}{ At 6 months } \\
\hline BMP & $40(27.4)$ & $89(61.0)$ & $17(10.3)$ & $2(1.4)$ \\
\hline Non-BMP & $10(10.1)$ & $66(66.7)$ & $21(21.2)$ & $2(2.0)$ \\
\hline \multicolumn{5}{|l|}{ At 2 years } \\
\hline BMP & $123(91.8)$ & $9(6.7)$ & $0(0)$ & $2(1.5)$ \\
\hline Non-BMP & $72(77.4)$ & $17(18.3)$ & $2(2.2)$ & $2(2.2)$ \\
\hline
\end{tabular}

Values are presented as number (\%).

$\mathrm{BMP}$, bone morphogenic protein.

$(p=0.13)$. Solid bony fusion was defined as a lack of motion, hardware migration, fracture, or lucency on flexionextension radiographs, thus having achieved a Bridwell grade of 1-2 fusion on radiographic analysis. The BMP group attained a significantly higher rate of fusion at 6 months follow-up at $88.4 \%$ as compared to the non-BMP group at $76.8 \%(p=0.016)$. By 2 years, however, there was no longer any significant difference between the 2 groups, with BMP group at $98.5 \%$ and non-BMP group at 95.7\% (Fig. 2). However, a higher percentage of patients in the BMP group $(91.8 \%$ vs. $77.4 \%, p=0.005)$ achieved Bridwell grade 1 than grade 2 (Table 3). Two patients in both the BMP group (1.5\%) and non-BMP group (2.2\%) were Bridwell grade 4 , while two patients in the non-BMP group (2.2\%) and none in the BMP group was Bridwell grade 3 at 2 years.

There were 12 cases of complication $(11.5 \%)$ in the non-
BMP group and $9(6.1 \%)$ in the BMP group (Table 4). The non-BMP group required 5 reoperations (4.8\%). Three patients required an operation for symptomatic nonunion of which 2 were Bridwell grade 4 and one Bridwell grade 3 at 2 years. One patient with cage migration required revision TLIF. One patient required revision for debridement of infection and another to revise a symptomatic medially placed pedicle screw. In the BMP group, 2 patients required reoperation (1.4\%) for nonunion, with both being Bridwell grade 4 at 2 years. Other adverse events in the BMP group included radiculitis $(\mathrm{n}=2)$, bone overgrowth in the foramen $(n=2)$, cage retropulsion $(n=2)$, and osteolysis $(n=1)$. All these patients were treated non-operatively with oral medications with eventual resolution of symptoms. Patients in both groups who required surgical revision eventually achieved good union with mild residual symptoms. 
Table 4. Complications and treatment in non-BMP and BMP group

\begin{tabular}{|c|c|c|}
\hline No. & Complication/adverse event & Result/treatment \\
\hline \multicolumn{3}{|c|}{ Non-BMP } \\
\hline 1 & Iliac crest wound infection+spine wound seroma & $\begin{array}{l}\text { Reoperation: debridement of iliac crest wound } \rightarrow \text { healed; } \\
\text { seroma resolved without tx }\end{array}$ \\
\hline 2 & Screw breakage & Asymptomatic \\
\hline 1 & $\begin{array}{l}\text { L5 screw medial penetration causing radicular } \\
\text { symptoms }\end{array}$ & Reoperation: screw revision \\
\hline 1 & Intraoperative cage fracture & Asymptomatic $\rightarrow$ fused \\
\hline 1 & Intraoperative cage malposition & Observed $\rightarrow$ fused at $2 \mathrm{yr}$ \\
\hline \multirow[t]{2}{*}{2} & Cage retropulsion & Reoperation: revised to open TLIF \\
\hline & & Treated conservatively \\
\hline \multirow[t]{4}{*}{4} & Nonunion & Reoperation: revised to open TLIF \\
\hline & & $\begin{array}{l}\text { Reoperation: revised to open TLIF, also has infected illiac } \\
\text { crest wound requiring debridement }\end{array}$ \\
\hline & & $\begin{array}{l}\text { Reoperation: infected non-union debrided and revision } \\
\text { fusion performed }\end{array}$ \\
\hline & & Asymptomatic $\rightarrow$ fibrous union \\
\hline \multicolumn{3}{|c|}{ BMP } \\
\hline 2 & Bone overgrowth with $\mathrm{LL}$ radiculopathy & Resolved with conservative treatment \\
\hline 2 & Cage retropulsion & Treated conservatively \\
\hline 2 & Radiculitis & Resolved with steroid course \\
\hline 1 & Osteolysis & Pain resolved with conservative treatment \\
\hline \multirow[t]{2}{*}{2} & Non-union & $\begin{array}{l}\text { Reoperation: revised with MIS reinstrumentation } \rightarrow \\
\text { eventual fusion }\end{array}$ \\
\hline & & Reoperation: revised to open TLIF \\
\hline
\end{tabular}

BMP, bone morphogenic protein; TLIF, transforaminal lumbar interbody fusion; MIS, minimally invasive surgery.

Preoperatively, out of 36 sexually active males, 10 (27.8\%) had some degree of ED preoperatively. Of the remaining 26 patients who did not have ED prior to surgery, $18(69 \%)$ answered the questionnaire at 6 months and 20 answered at 24 months (83\%). Two of 11 patients (18.2\%) in the BMP group and one of 9 patients (11.1\%) in the non-BMP group had ED at 6 months. These 3 patients continued to have ED at 24 months with no additional patients.

\section{Discussion}

MIS of the spine is thought to decrease postoperative pain and allow quicker recovery by limiting soft-tissue retraction and dissection. However, the smaller quantity of available local bone graft in the MIS approach can necessitate the use of bone graft and/or graft substitutes to ensure bony arthrodesis. BMPs stimulate bone formation in extra-osseous tissues without the inclusion of any other biologically active proteins $[6,11,19]$ as well as increase fusion rate while avoiding donor site morbidity. A metaanalysis involving 10 randomized controlled trials that compared BMP and iliac crest bone graft showed that rhBMP-2 was superior to the graft in achieving fusion success and avoiding reoperation [7]. Despite the BMP group being significantly older and having more multilevel surgery than the non-BMP group in our study, the BMP group displayed a higher fusion rate at 6 months. There was no difference in fusion rate at 2 years or the symptomatic nonunion rate requiring eventual revision surgery between the two groups. Thus, BMP appears to help in achieving earlier fusion as compared to the non- 


\section{BMP group in MIS-TLIF.}

Several studies have suggested that the use of rhBMP-2 is not associated with a superior clinical outcome, including a meta-analysis of reviewed 13 randomized controlled trials and 31 cohort studies of rhBMP-2 versus any controlled and uncontrolled studies of harms $[7,9,14,20]$. However, another study reported improved clinical scores with BMP use at 1 and 6 months after surgery [21]. In our study, BMP patients had lower mean total morphine usage $(8 \mathrm{mg})$ in the immediate postoperative period when compared to the non-BMP group. One possible explanation may be an anti-inflammatory effect of BMP for these patients who were significantly older as well has having longer fusion levels [22]. At 6 months, patients in the BMP group had lower back and leg pain, which may be explained by earlier fusion in this group compared to the non-BMP group. Patients in the BMP group also had better VAS score for leg pain and NASS score, and a marginally statistically significant difference in ODI score at 2 years. But, there was however no statistically significant difference in the SF-36 scores at 6 months and 2 years. BMP was not inferior to other forms of bone graft in terms of pain scores or functional outcome at 6 months and 2 years. The improvements may be partly attributable to the earlier rate of fusion in the BMP group as compared to the non-BMP group. We are unsure whether a higher proportion of patients achieving Bridwell grade 1 than grade 2 in the BMP group had any contribution to better leg pain, ODI, and NASS scores at 2 years.

Although the YODA study described the potential complications associated with the use of BMP [9], other studies have showed evidence of minimal complications related to the use of rhBMP-2. A study involving 55,862 cases of spinal fusion with and without BMP concluded that the use of BMP was not associated with more complications in thoracolumbar and posterior cervical fusions [23]. Another study of 1,037 patients who underwent posterolateral fusion with rhBMP-2 noted few complications directly attributable to rhBMP-2 [24]. A recent study reported that the occurrence of BMP-related complications, such as seroma and ectopic bone, were rare [25]. The incidence of BMP-related complications in our study was low (4.8\%) and all resolved with conservative treatment. There was also no increased incidence of ED in patients with BMP. It may be that an anterior approach, rather than BMP alone, may have a greater role in the production of ED. Several studies looking at cancer and BMP have yielded conflict- ing results [26,27]. Our study does not provide illumination, as the follow-up was too short.

There are some limitations in our study. It is an observational cohort comparison study and not a randomized controlled trial so unknown confounding factors may not have been accounted for. There could be a selection bias as patients could select treatment. However, the demographics of both patients groups are similar (except age) and a single surgeon performed the procedures, which limited any intraoperative technical differences. For the non-BMP cases, iliac crest graft and/or demineralized bone matrix were used to facilitate fusion depending on surgeon preference. As there was no uniform or standard graft for all cases, no actual comparison between recombinant human bone morphogenic protein-2 versus autogenous iliac crest bone graft or demineralized bone matrix was made. It is normative and acceptable to use such materials in cases where BMP is not used in patients with MIS surgery due to the lack of autograft. The type of cage and instrumentation was not standardized but we believe the attributable difference should be minimal as the surgical technique was consistent. Flexion/extension standing lateral radiographs were used to assess fusion instead of a computed tomography scan.

\section{Conclusions}

BMP used to augment fusion in MIS-TLIF is an acceptable alternative to autogenous iliac crest graft and/or demineralized bone matrix, with potential benefit of less pain in the early and intermediate postoperative followup. This may partly be due to earlier and more solid fusion in the BMP group. The complication rate from BMP is low and resolution of BMP-associated symptoms usually occurs with conservative measures.

\section{Conflict of Interest}

No potential conflict of interest relevant to this article was reported.

\section{References}

1. Mannion RJ, Nowitzke AM, Wood MJ. Promoting fusion in minimally invasive lumbar interbody stabilization with low-dose bone morphogenic protein-2: but what is the cost? Spine J 2011;11:527-33. 
2. Peng CW, Yue WM, Poh SY, Yeo W, Tan SB. Clinical and radiological outcomes of minimally invasive versus open transforaminal lumbar interbody fusion. Spine (Phila Pa 1976) 2009;34:1385-9.

3. Kim CW, Siemionow K, Anderson DG, Phillips FM. The current state of minimally invasive spine surgery. J Bone Joint Surg Am 2011;93:582-96.

4. Eck JC, Hodges S, Humphreys SC. Minimally invasive lumbar spinal fusion. J Am Acad Orthop Surg 2007; 15:321-9.

5. Seng C, Siddiqui MA, Wong KP, et al. Five-year outcomes of minimally invasive versus open transforaminal lumbar interbody fusion: a matched-pair comparison study. Spine (Phila Pa 1976) 2013;38:204955.

6. Whang PG, Wang JC. Fusion biology and contemporary graft options. Semin Spine Surg 2003;15:460-72.

7. Chen Z, Ba G, Shen T, Fu Q. Recombinant human bone morphogenetic protein-2 versus autogenous iliac crest bone graft for lumbar fusion: a meta-analysis of ten randomized controlled trials. Arch Orthop Trauma Surg 2012;132:1725-40.

8. Fischer CR, Cassilly R, Cantor W, Edusei E, Hammouri Q, Errico T. A systematic review of comparative studies on bone graft alternatives for common spine fusion procedures. Eur Spine J 2013;22:142335.

9. Simmonds MC, Brown JV, Heirs MK, et al. Safety and effectiveness of recombinant human bone morphogenetic protein-2 for spinal fusion: a meta-analysis of individual-participant data. Ann Intern Med 2013; 158:877-89.

10. Hsu WK, Wang JC. The use of bone morphogenetic protein in spine fusion. Spine J 2008;8:419-25.

11. Carlisle E, Fischgrund JS. Bone morphogenetic proteins for spinal fusion. Spine J 2005;5(6 Suppl):240S249S.

12. Carragee EJ, Hurwitz EL, Weiner BK. A critical review of recombinant human bone morphogenetic protein-2 trials in spinal surgery: emerging safety concerns and lessons learned. Spine J 2011;11:47191.

13. Even J, Eskander M, Kang J. Bone morphogenetic protein in spine surgery: current and future uses. J Am Acad Orthop Surg 2012;20:547-52.

14. Fu R, Selph S, McDonagh M, et al. Effectiveness and harms of recombinant human bone morphogenetic protein-2 in spine fusion: a systematic review and meta-analysis. Ann Intern Med 2013;158:890-902.

15. Singh K, Nandyala SV, Marquez-Lara A, et al. Clinical sequelae after rhBMP-2 use in a minimally invasive transforaminal lumbar interbody fusion. Spine J 2013;13:1118-25.

16. Chrastil J, Low JB, Whang PG, Patel AA. Complications associated with the use of the recombinant human bone morphogenetic proteins for posterior interbody fusions of the lumbar spine. Spine (Phila Pa 1976) 2013;38:E1020-7.

17. Bridwell KH, Lenke LG, McEnery KW, Baldus C, Blanke K. Anterior fresh frozen structural allografts in the thoracic and lumbar spine: do they work if combined with posterior fusion and instrumentation in adult patients with kyphosis or anterior column defects? Spine (Phila Pa 1976) 1995;20:1410-8.

18. Lee KH, Yue WM, Yeo W, Soeharno H, Tan SB. Clinical and radiological outcomes of open versus minimally invasive transforaminal lumbar interbody fusion. Eur Spine J 2012;21:2265-70.

19. Jeong GK, Sandhu HS. Applications of recombinant human bone morphogenetic protein-2 (rhBMP-2) in spinal surgery. Semin Spine Surg 2006;18:15-21.

20. Michielsen J, Sys J, Rigaux A, Bertrand C. The effect of recombinant human bone morphogenetic protein-2 in single-level posterior lumbar interbody arthrodesis. J Bone Joint Surg Am 2013;95:873-80.

21. Slosar PJ, Josey R, Reynolds J. Accelerating lumbar fusions by combining rhBMP-2 with allograft bone: a prospective analysis of interbody fusion rates and clinical outcomes. Spine J 2007;7:301-7.

22. Zhang X, Zhang Z, Shen G, Zhao J. Enhanced osteogenic activity and anti-inflammatory properties of Lenti-BMP-2-loaded $\mathrm{TiO}(2)$ nanotube layers fabricated by lyophilization following trehalose addition. Int J Nanomedicine 2016;11:429-39.

23. Williams BJ, Smith JS, Fu KM, et al. Does bone morphogenetic protein increase the incidence of perioperative complications in spinal fusion? A comparison of 55,862 cases of spinal fusion with and without bone morphogenetic protein. Spine (Phila Pa 1976) 2011;36:1685-91.

24. Glassman SD, Howard J, Dimar J, Sweet A, Wilson G, Carreon L. Complications with recombinant human bone morphogenic protein-2 in posterolateral spine fusion: a consecutive series of 1037 cases. Spine (Phila 
Pa 1976) 2011;36:1849-54.

25. Crandall DG, Revella J, Patterson J, Huish E, Chang M, McLemore R. Transforaminal lumbar interbody fusion with rhBMP-2 in spinal deformity, spondylolisthesis, and degenerative disease: part 1: Large series diagnosis related outcomes and complications with 2- to 9-year follow-up. Spine (Phila Pa 1976) 2013;38:1128-36.
26. Langenfeld EM, Langenfeld J. Bone morphogenetic protein-2 stimulates angiogenesis in developing tumors. Mol Cancer Res 2004;2:141-9.

27. Cooper GS, Kou TD. Risk of cancer after lumbar fusion surgery with recombinant human bone morphogenic protein-2 (rh-BMP-2). Spine (Phila Pa 1976) 2013;38:1862-8. 